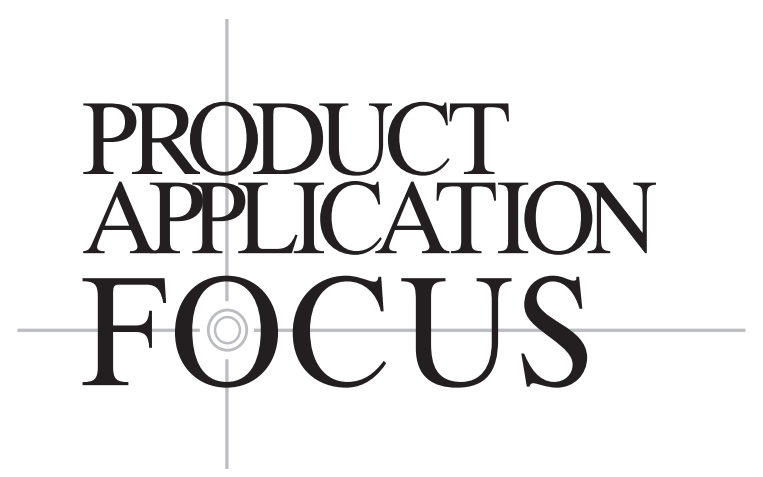

\title{
Minor Groove Binder-Conjugated DNA Probes for Quantitative DNA Detection by Hybridization-Triggered Fluorescence
}

\author{
I.A. Afonina, M.W. Reed, E. Lusby, I.G. Shishkina, and Y.S. Belousov \\ Epoch Biosciences, Bothell, WA, and Synthetic Genetics, San Diego, CA, USA
}

BioTechniques 32:940-949 (April 2002)

\begin{abstract}
Here we describe the properties of a novel class of oligonucleotide probes capable of sensitive hybridization-triggered fluorescence. These fluorogenic probes, known commercially as MGB Eclipse ${ }^{T M}$ probes, are characterized by having a conjugated minor groove binder (MGB) ligand at the 5'-end and a fluorophore at the 3'-end. Additionally, they have an efficient quencher moiety at the 5'end that is useful with a wide variety of fluorescent dyes. Fluorescence of the single-stranded MGB Eclipse ${ }^{T M}$ probe is efficiently quenched by the interaction of the terminal dye and quencher groups when not hybridized. Upon hybridization to a complementary target, the MGB molecule folds into duplex and hyper-stabilizes it, allowing the use of shorter, more specific probe sequences. The 5'-MGBquencher group also prevents nuclease digestion by Taq DNA polymerase during PCR. Because of the hybridization-triggered fluorescence and the excellent specificity imparted by the $M G B$, these 5'-MGB Eclipse probes have great versatility for real-time PCR applications. The high sensitivity and specificity are illustrated using single nucleotide polymorphism detection, viral load determination, and gene expression analysis.
\end{abstract}

\section{INTRODUCTION}

Real-time DNA target detection during amplification has proven to be a robust and efficient method for sensitive and quantitative sequence detection and identification. Amplification and hybridization, when combined in one step, can be performed in a fully automated, high-throughput, closed-tube format. Methods used to date for this technique are based primarily on using fluorescence resonance energy transfer (FRET) probes that are designed to hybridize to an internal region of a PCR product during the annealing stage. These probes are either cleaved in the reaction (as Taqman ${ }^{\circledR}$ MGB probes) or undergo a conformation change in the presence of a complementary DNA target (as Molecular Beacons) and both utilize a disruption of FRET, a phenomenon described by Parkhurst and Parkhurst (18) and recently reviewed by Didenko (6).

For Taqman MGB probes, the 5 '-exonuclease activity of Taq DNA polymerase cleaves the probe from the $5^{\prime}$-end, between the fluorophore and quencher, thus releasing fluorescence (11). Modified bases (9) and minor groove binder (10) reportedly improve the performance of the Taqman MGB probes. Fluorescence quenching of a beacon probe is a result of an intramolecular stem formation that brings the fluorophore and quencher in close proximity (22). When hybridized to the target DNA strand, quencher and fluorophore are separated enough to produce a fluorescent signal. The beacon probes are 35-40 bases long, including the required extra 10-12 bases for hairpin formation. Because of the need to balance the stabilities of the stem-loop with the hybridized probe, the design of such probes can be complicated.

Methods that use double-strand-specific fluorophores such as $\mathrm{SYBR}^{\circledR}$ Green (20) or labeled primers (15) can take advantage of less expensive reagents but do not provide assurance of specific amplification. To prove that signal increase is not due to false priming or primer-dimer formation, post-PCR steps such as a product melting curve analysis are required. The sequence specificity provided by fluorogenic probes such as Taqman MGB or Molecular Beacons is beneficial for highthroughput applications. Here we describe a third type of fluorogenic probe for DNA measurement that we call MGB Eclipse $^{\mathrm{TM}}$ probes.

We have been exploring the chemistry and applications of oligonucleotides with a conjugated minor groove binder (MGB) ligand, which form hyper-stabilized duplexes with 
complementary DNA $(1,2,10,12)$. The sequence specificity of these novel agents provides a significant advantage for hightemperature applications such as PCR (10). We have also developed a non-fluorescent quencher (the Eclipse Dark Quencher, Q) for use in our fluorogenic MGB Eclipse probes (13). When used as hybridization probes, the Eclipse Dark Quencher combination obviates the need for the stem structure, as in Molecular Beacons, and allows the use of much shorter oligonucleotides. Here we report the synthesis and properties of MGB Eclipse oligonucleotides with an attached fluorophore and describe their performance as hybridization-triggered fluorogenic probes for PCR. The short length and unique structure of MGB Eclipse probes give very low fluorescent background and excellent specificity, and this performance is demonstrated in the real-time PCR applications of single nucleotide polymorphism (SNP) detection, viral load determination, and gene expression analysis.

\section{MATERIALS AND METHODS}

\section{Templates}

Human genomic DNAs from the pedigree family no. 66 were used to study the RRM1 SNP $(16,17)$. Allele 1 homozygous DNA (mother), allele 2 homozygous DNA, and heterozygous DNA (son) were purchased from Coriell Institute of Medical Research. Other human genomic DNAs used in the SNP assay were purchased from the same institute, with the exception of the "p53" and "CYP2D6*4" templates. Genotyping was done previously in our laboratory using restriction length polymorphism (14) and Taqman MGB methods. PCMV-p53 and pCMV-p53mt135 plasmids containing wild-type and mutant p53G1017A constructs were from BD Biosciences Clontech (Palo Alto, CA, USA). CYP2D6*4 wild-type and mutant human genomic DNAs were a gift from Dr. Ed Lusby (Synthetic Genetics, San Diego, CA, USA).

cDNA template for gene expression analysis of human cellular oncogene c-fos (24) was created by using total RNA from human placenta (supplied by BD Biosciences Clontech). Reverse transcription was performed using an Advantage $^{\mathrm{TM}}$ RT-for-PCR kit as recommended by BD Biosciences Clontech.

DNA fragment containing a partial sequence of glycoprotein D gene (19) of herpes simplex virus (HSV) type 1 was derived from DNA isolated from infected cells (strain $\mathrm{F}$ from ATCC no. VR-733) by PCR (23). The PCR fragment was gelpurified and quantitated by UV absorption.

\section{Oligodeoxyribonucleotides}

Table 1 shows the sequences of oligonucleotides used in the current publication. MGB Eclipse probes were designed using a $T_{m}$ prediction program created for $3^{\prime}$-MGB Taqman probes (Primer Express ${ }^{\circledR}$ software; Applied Biosystems, Foster City, CA, USA). A complementary strand was used to approximate the increase in stability due to the $5^{\prime}$-MGB in MGB Eclipse probes.

Primers were synthesized using standard phosphoramidite chemistry. The MGB Eclipse probes were prepared by automated DNA synthesis on a MGB-Q-modified glass support (manuscript in preparation) using $5^{\prime}$ - $\beta$-cyanoethyl phosphoramidites (Glen Research, Sterling, VA, USA) designed for the synthesis of oligonucleotide segments in the $5^{\prime} \rightarrow 3^{\prime}$ direction. Oligonucleotide synthesis was performed on an ABI PRISM $^{\circledR} 394$ synthesizer (Applied Biosystems), according to the protocol supplied by the manufacturer, using a $0.02 \mathrm{M}$ iodine solution. 6-carboxyfluorescein (FAM) and 6-carboxy$4,7,2^{\prime}, 7^{\prime}$-tetrachlorofluorescein (TET) reporting dyes were introduced at the last step of the synthesis using the corresponding phosphoramidites (Glen Research). A Molecular Beacon corresponding to RRM1 allele 1 SNP was prepared using standard methods. It was designed with the help of DNA-fold software (http://bioinfo.math.rpi.edu/ mfold/dna) based on the work of the Zucker laboratory (21). The 3'MGB-Q Taqman MGB probe corresponding to RRM1 allele 1 SNP was synthesized on the same type of Q-MGB solid support that was used for the preparation of the MGB Eclipse probes. Standard 5'-DMT phosphoramidites were used. It was designed with the help of the Primer Express software. All oligonucleotides were purified by reverse-phase HPLC.

\section{Radioactive Labeling of the Oligonucleotides}

To monitor the digestion of Taqman MGB, Molecular Beacon, and MGB Eclipse probes by Taq DNA polymerase during $\mathrm{PCR}$, the corresponding oligonucleotides were labeled with ${ }^{32} \mathrm{P}$. The $5^{\prime}$-ends of the Taqman and Molecular Beacon probes were phosphorylated with $\mathrm{T} 4$ polynucleotide kinase (Promega, Madison, WI, USA) and $\left[\gamma_{-}{ }^{32} \mathrm{P}\right] \mathrm{ATP}(5000$ $\mathrm{Ci} / \mathrm{mmol}, 10 \mathrm{mCi} / \mathrm{mL}$; Amersham Biosciences, Piscataway, NJ, USA). The $3^{\prime}$-end of the MGB Eclipse probe was labeled with terminal deoxynucleotidyl transferase (Promega) and $[\alpha-$ $\left.{ }^{32} \mathrm{P}\right]$ cordycepin triphosphate (Perkin Elmer Life Sciences).

\section{Real-Time PCR}

Real-time PCR was conducted in either a ABI PRISM 7700 or a LightCycler ${ }^{\mathrm{TM}}$ LC 24 (Idaho Technology) thermal cycling fluorimeter.

On the ABI PRISM 7700 instrument, we pefromed $50 \mathrm{cy}$ cles of three-step PCR $\left(95^{\circ} \mathrm{C}\right.$ for $30 \mathrm{~s}, 56^{\circ} \mathrm{C}$ for $30 \mathrm{~s}$, and $76^{\circ} \mathrm{C}$ for $30 \mathrm{~s}$ ) after $2 \mathrm{~min}$ at $50^{\circ} \mathrm{C}$ and $2 \mathrm{~min}$ at $95^{\circ} \mathrm{C}$. The annealing temperature was sometimes elevated for the mismatch detection, as indicated in the Results section. The reactions contained $0.25 \mu \mathrm{M}$ MGB Eclipse probe, $100 \mathrm{nM}$ primer complementary to the same strand as the probe, $4 \mu \mathrm{M}$ opposite strand primer, $125 \mu \mathrm{M}$ dATP, $125 \mu \mathrm{M}$ dCTP, $250 \mu \mathrm{M}$ dUTP, 0.25 U JumpStart ${ }^{\mathrm{TM}}$ Taq DNA polymerase (Sigma, St. Louis, MO, USA), 0.125 U AmpErase ${ }^{\circledR}$ Uracil $N$-glycosylase (Applied Biosystems) in $1 \times \mathrm{PCR}$ buffer $(20 \mathrm{mM}$ Tris- $\mathrm{HCl}, \mathrm{pH}$ $8.7,50 \mathrm{mM} \mathrm{NaCl}, 5 \mathrm{mM} \mathrm{MgCl}_{2}$ ) in a $10-\mu \mathrm{L}$ reaction. For the $25-\mu \mathrm{L}$ reaction, the amounts of the enzymes were adjusted proportionally. AmpErase uracil $N$-glycosylase was used with dUTP (instead of TTP) in all reactions as a PCR product carryover prevention measure. The amount of the added template is indicated in the Results section. The increase in fluorescent signal was registered during the annealing step of the reaction.

For the PCR in the LightCycler, the PCR mixture was the same, with the exception that non-acetylated BSA was added to a final concentration of $250 \mu \mathrm{g} / \mu \mathrm{L}$. Cycling was performed as follows: $2 \mathrm{~min}$ at $50^{\circ} \mathrm{C}, 1 \mathrm{~min}$ at $95^{\circ} \mathrm{C}$, followed by $50 \mathrm{cy}-$ cles of $0 \mathrm{~s}$ at $95^{\circ} \mathrm{C}, 20 \mathrm{~s}$ at $56^{\circ} \mathrm{C}$, and $30 \mathrm{~s}$ at $76^{\circ} \mathrm{C}$. The final concentration of fluorogenic probes (MGB Eclipse, Molecular Beacon, and Taqman MGB) was $250 \mathrm{nM}$. Primer concentrations for the MGB Eclipse probes were the same as for the 
Table 1. Sequence of Primers and Fluorogenic Probes

\begin{tabular}{|c|c|c|c|}
\hline & $5^{\prime}$-end & Sequence & 3'-end \\
\hline \multicolumn{4}{|c|}{ RRM1 T1037G mutation; accession no. XM 006065} \\
\hline Primer 1 (sense) & & ATG GCC TTG TAC CGA TGC TGA & \\
\hline Primer 2 (antisense) & & GTA CTT TCA ATT CAT GGA GCA TAC CT & \\
\hline MGB Eclipse probe allele 1 (sense) & MGB-Q & ATA TCT AGC TGT GTT GA & FAM \\
\hline MGB Eclipse probe allele 2 (sense) & MGB-Q & ATA TCG AGC TGT GTT GA $A^{b}$ & TET \\
\hline Molecular Beacon allele 1 (sense) & FAM & cca acc AT CCA CAT ATC TAG CTG TGT TG ggt tgga & Dabcyl \\
\hline Taqman MGB allele 1 (sense) & FAM & ATA TCT AGC TGT GTT G & MGB-Q \\
\hline \multicolumn{4}{|c|}{ CYP2D6*4 C4495T mutation accession no. M33388 } \\
\hline Primer 1 (sense) & & GCC GCC TTC GCC AAC CAC & \\
\hline Primer 2 (antisense) & & GGA GGC GAT CAC GTT GCT CAC G & \\
\hline MGB Eclipse probe allele 1 (antisense) & MGB-Q & GTC CTG G^GG^GTG^ & FAM \\
\hline MGB Eclipse probe allele 2 (antisense) & MGB-Q & GTC TTG $G^{\wedge} G G^{\wedge} G T G \wedge$ & TET \\
\hline \multicolumn{4}{|c|}{ HFE C282Y mutation accession no. U91328 } \\
\hline Primer 1 (sense) & & GGC TGG ATA ACC TTG GCT GT & \\
\hline Primer 2 (antisense) & & CAG ATC ACA ATG AG^G GGC TG & \\
\hline MGB Eclipse probe allele 1 (antisense & MGB-Q & CAC CTG GCA CGT ATA T & FAM \\
\hline MGB Eclipse probe allele 2 (antisense & MGB-Q & CTG GTA CGT ATA TCT CT & TET \\
\hline \multicolumn{4}{|c|}{ Factor II G20210A mutation; accession nos. M1762 and M33691 } \\
\hline Primer 1 (sense) & & TCC TGG AAC CAA TCC CGT GAA A & \\
\hline Primer 2 (antisense) & & GCT GCC CAT GAA TAG CAC TG & \\
\hline MGB Eclipse probe allele 1 (antisense) & MGB-Q & TTG AGG CTC GCT GA & FAM \\
\hline MGB Eclipse probe allele 2 (antisense) & MGB-Q & TTG AGG CTT GCT GA & TET \\
\hline \multicolumn{4}{|c|}{ MTHFR C677T mutation accession no. XM_030156 } \\
\hline Primer 1 (sense) & & CTG ACC TGA AGC ACT TGA AGG AG & \\
\hline Primer 2 (antisense) & & GCG GAA GAA TGT GTC AGC CTC AAA G & \\
\hline MGB Eclipse probe allele 1 (antisense) & MGB-Q & ATC GGC TCC CGC & FAM \\
\hline MGB Eclipse probe allele 2 (antisense) & MGB-Q & A ATC GAC TCC CGC & TET \\
\hline \multicolumn{4}{|l|}{ p53 G1017A mutation } \\
\hline Primer 1 (sense) & & CAA GTC TGT GAC TTG CA & \\
\hline Primer 2 (antisense) & & TGT GGA ATC AAC CCA CAG & \\
\hline MGB Eclipse probe allele 1 (antisense & MGB-Q & TCA GTT GGC AAA ACA TC & FAM \\
\hline \multicolumn{4}{|l|}{ c-myc; accession no. J00120 } \\
\hline Primer 1 (sense) & & GGA AAA CCA GCA GCC TCC CG & \\
\hline Primer 2 (antisense) & & GTT CTC CTC CTC GTC GCA GT & \\
\hline MGB Eclipse probe allele 1 (sense) & MGB-Q & AGC TTC ACC AAC AGG A & FAM \\
\hline \multicolumn{4}{|l|}{ c-fos; accession no. V01512 } \\
\hline Primer 1 (sense) & & TCC AAG CGG AGA CAG AC & \\
\hline Primer 2 (antisense) & & GCA GGC AGG TCG GTG AG & \\
\hline MGB Eclipse probe (antisense) & MGB-Q & CTT CAG CAG GTT GGC AAT & FAM \\
\hline \multicolumn{4}{|l|}{ HSV 1,2; accession no. AF333383 } \\
\hline Primer 1 (sense) & & ATC CGA ACG CAG CCC CGC TG & \\
\hline Primer 2 (antisense) & & TCT CCG TCC AGT CGT TTA TCT TC & \\
\hline MGB Eclipse probe (antisense) & MGB-Q & CCC AGG TTA TCC TCG CT & FAM \\
\hline
\end{tabular}


ABI PRISM 7700. The primer concentration for the Molecular Beacon and Taqman MGB probes was $0.50 \mu \mathrm{M}$ each. Fluorescence was registered during the annealing and denaturing steps of the reaction.

Results were taken directly from the screen, or clipped files were transferred to Microsoft ${ }^{\circledR}$ Excel $^{\circledR}$, as instructed by the manufacturers.

\section{PCR with Radioactive Probes}

PCR conditions with radioactive probes were the same as for the real-time PCR, with the exception that the control reactions did not contain Taq DNA polymerase. After completion of the PCR, aliquots of each sample were separated on a denaturing polyacrylamide gel. Phosphor imaging was used to estimate the degradation of the probes.

\section{Real-Time RT-PCR}

Real-time RT-PCR was done either in two steps (RT and PCR in different tubes) or in one step (RT and PCR combined). Two-step RT-PCR was performed using the Clontech Advantage RT-for-PCR kit. Total RNA from human placenta and random primers were supplied by BD Biosciences Clontech. One microliter of a completed RT reaction was used as a template in $10 \mu \mathrm{L}$ real-time PCR with appropriate primers and FAM-labeled MGB Eclipse probe as described earlier.

The one-tube reaction was performed using the QIAGEN ${ }^{\circledR}$ OneStep RT-PCR Kit (Qiagen, Valencia, CA, USA) with different amounts of total RNA from human placenta (1-100 ng) per reaction. We followed the protocol suggested by the manufacturer with minor exceptions. Instead of the recommended $0.6 \mu \mathrm{M}$ each gene-specific primer, $2-4 \mu \mathrm{M}$ antisense primer and $0.1 \mu \mathrm{M}$ sense primer were used. The MGB Eclipse probe (sense) was added to a final concentration of $0.2 \mu \mathrm{M}$ to enable real-time detection. RNase inhibitor was used at $5 \mathrm{U} /$ reaction. Thermal cycling conditions were within the recommended range and included $30 \mathrm{~min}$ at $50^{\circ} \mathrm{C}$ for $\mathrm{RT}, 15 \mathrm{~min}$ at $95^{\circ} \mathrm{C}$ for the initial PCR activation step, and 40 three-step cycles of denaturation for $30 \mathrm{~s}$ at $94^{\circ} \mathrm{C}$, annealing for $30 \mathrm{~s}$ at $56^{\circ} \mathrm{C}$, and extension for $30 \mathrm{~s}$ at $76^{\circ} \mathrm{C}$. Extension time was reduced to 30 s from 1 min on the ABI PRISM 7700 because of the small size of the PCR products. The extension temperature was elevated from $72^{\circ} \mathrm{C}$ to $76^{\circ} \mathrm{C}$ to ensure that all Eclipse probes are melted off the growing DNA strand and do not arrest PCR. The final extension step was omitted. Fluorescent readings were taken at the annealing stage of the reaction.

\section{RESULTS AND DISCUSSION}

\section{Fluorescent Properties of MGB Eclipse Probes}

Figure 1A shows the structure of the MGB Eclipse probe. The $5^{\prime}$-end of the MGB Eclipse Probes is conjugated to a crescent-shaped minor groove binder conjugated via a linker that also bears the Eclipse Dark Quencher. The fluorescent dye is attached to the $3^{\prime}$-end of the probe. These probes are prepared by $5^{\prime} \rightarrow 3^{\prime}$ oligonucleotide synthesis using a solid support that carries the MGB-Q moiety. When an MGB Eclipse probe is not bound to the target, the fluorophore is quenched because the probe assumes a random coil structure in solution. When hybridized to the target, the quencher and the fluorophore are spatially separated, and the probe emits fluorescence, as shown schematically in Figure 1B.

The conjugated MGB ligands are derivatives of naturally occurring antibiotics that fit into the minor groove of the helix formed by dsDNA (8). The hydrophobic binding of the MGB to the DNA helix increases the $\mathrm{T}_{\mathrm{m}}$ by $15^{\circ} \mathrm{C}-30^{\circ} \mathrm{C}$, which allows the use of shorter probes with high specificity (10). This property is particularly beneficial in the design of probes for SNP detection and for probes to short, conserved sequences of viral genomes or multi-gene families. An especially useful feature of the conjugated MGB is that it stabilizes A-T hydrogen bonds more than G-C bonds. This in turn stabilizes weaker sequences, thereby reducing the influence of target sequence on the $T_{m}(12)$. In general, the $T_{m}$ of short probes (10-15 nucleotides) can be made almost sequence-independent by adding the MGB.

The novel Eclipse Dark Quencher quenches the fluores-
A

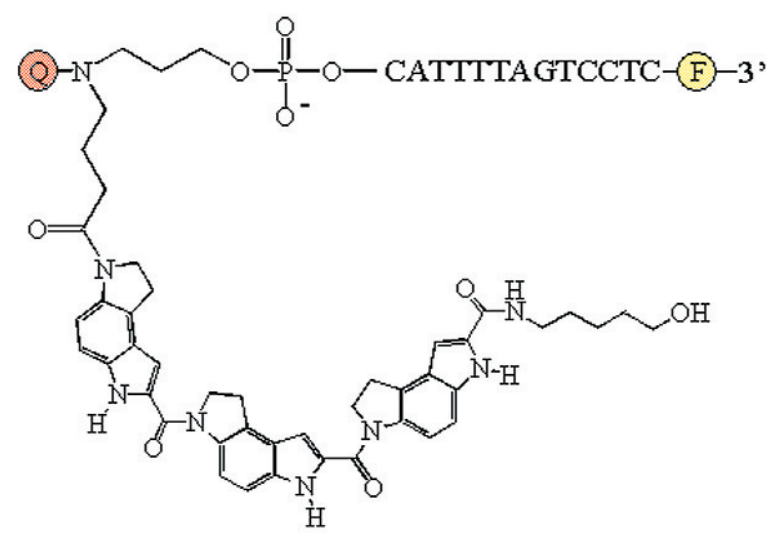

B

MGB Eclipse ${ }^{\text {TM }}$ Probe

Quenched Probe

(F) = Fluorescent Group

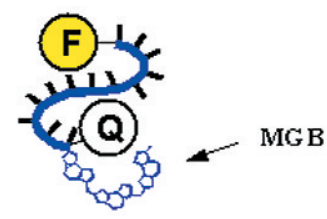

Q = Quencher Group

Fluorescent Probe

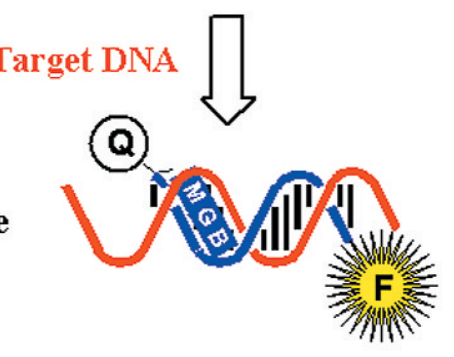

Figure 1. MGB Eclipse probe detection system. (A) Structure of MGB Eclipse probe. (B) Detection system. 
cence of a wide range of dyes with emission maxima from 400 to $650 \mathrm{~nm}$ (data not shown), in contrast to the 360-560 $\mathrm{nm}$ range of the widely used Dabcyl (4-(4-dimethylaminophenylazo)benzoyl-) group (13). The Eclipse Dark Quencher and MGB work in concert to eliminate fluorescence for non-hybridized probe molecules while allowing much stronger fluorescence for hybridized probes.

Fluorogenic probes used in real-time PCR rely on the detection and quantification of a fluorescent reporter, the signal of which increases because of a release of quenching caused by an event occurring as a result of, and in direct proportion to, the amount of PCR product in a reaction. Figure 2 illustrates the distinct patterns of fluorescent signals generated from three different fluorogenic probes during PCR. PCR was conducted in a LightCycler using RRM1-specific primers (Table 1) and fluorogenic probes (MGB Eclipse probe, Molecular Beacon, or Taqman MGB). Each reaction began with 10 ng "66 son" human genomic DNA as a template in a 10$\mu \mathrm{L}$ reaction. Fluorescent measurements were taken at the denaturing and annealing steps.

The fluorescence of the MGB Eclipse probe that was taken at the denaturing temperature is low and does not increase during PCR (Figure 2, Ai), indicating absence of probe digestion. The 5'-MGB-Q moiety effectively blocks nuclease digestion by Taq DNA polymerase. The fluorescence of the MGB Eclipse probe measured during the annealing step of PCR increases with time, which is indicative of the growing amount of target product to which the MGB Eclipse probes hybridize.

Molecular Beacons, like MGB Eclipse probes, are designed to remain intact during the amplification reaction and rebind to target in every cycle for signal measurement. The fluorescence of the Molecular Beacon, taken at the denaturing temperature, is high and increases about 20\% during PCR, indicating partial digestion of the probe. This "nuclease effect" depends on the probe sequence and conditions of the PCR and usually accounts for $10 \%-40 \%$ of the total signal. Molecular Beacon fluorescence measured during annealing step of the PCR increases with time in accordance with PCR product amount (Figure 2 , Bi). The fluorescent background of the MGB Eclipse probes is significantly lower than, and the signal-to-noise ratio comparable to, the much longer Molecular Beacon probes.

The fluorescence pattern of the Taqman MGB probe is different from the previous two probes (Figure 2, Ci). At the initial steps of the PCR, when there is no product to hybridize to, fluorescence of the probe is low at both $56^{\circ} \mathrm{C}$ and $95^{\circ} \mathrm{C}$. This is typical for all short MGB probes. With PCR progression, nuclease digestion leads to signal accumulation and an increase in fluorescence at both temperatures. At the last cycles of PCR, there is evidence of a "hybridization fluorescence effect" in the behavior of a Taqman MGB probe: fluorescence at $56^{\circ} \mathrm{C}$ is higher than at $95^{\circ} \mathrm{C}$. Presumably, the undigested fraction of the probe behaves like hybridization probes that fluoresce upon hybridization.

To support the evidence of the probe degradation (or lack of it) during PCR, we substituted the fluorescent label with a radioactive one (see Materials and Methods section). As expected, MGB Eclipse probes showed no traces of degradation after the completion of PCR (Figure 2, Aii). The Molecular Beacon probe was more than $30 \%$ digested by Taq DNA polymerase (Figure 2, Bii). Taqman MGB was almost completely digested (Figure 2, Cii). None of the probes was heatdegraded during PCR (see no enzyme controls in Figure 2, ii).

\section{SNP Detection}

We have developed MGB Eclipse probes that accurately discriminate SNPs. Coupling of MGB to an oligonucleotide greatly increases the stability of duplexes formed between oligonucleotide and target, allowing shorter probes to be used with improved mismatch discrimination (10). Figure 3 shows real-time plots from six pairs of MGB Eclipse probes labeled with FAM (one allele) and TET (another allele). Human genomic DNAs (25 ng) homozygous for each allele (and, when available, heterozygous) were used as templates in $25-\mu \mathrm{L}$ reactions. In case of $\mathrm{p} 53 \mathrm{G} 1017 \mathrm{~A}$ polymorphism, $10^{6}$ copies of the plasmid containing wild-type or mutant p53 constructs were added to the reaction. Primers were designed to produce products that are $80-200 \mathrm{bp}$ in length with minimal secondary structure formed between the primers and their respective

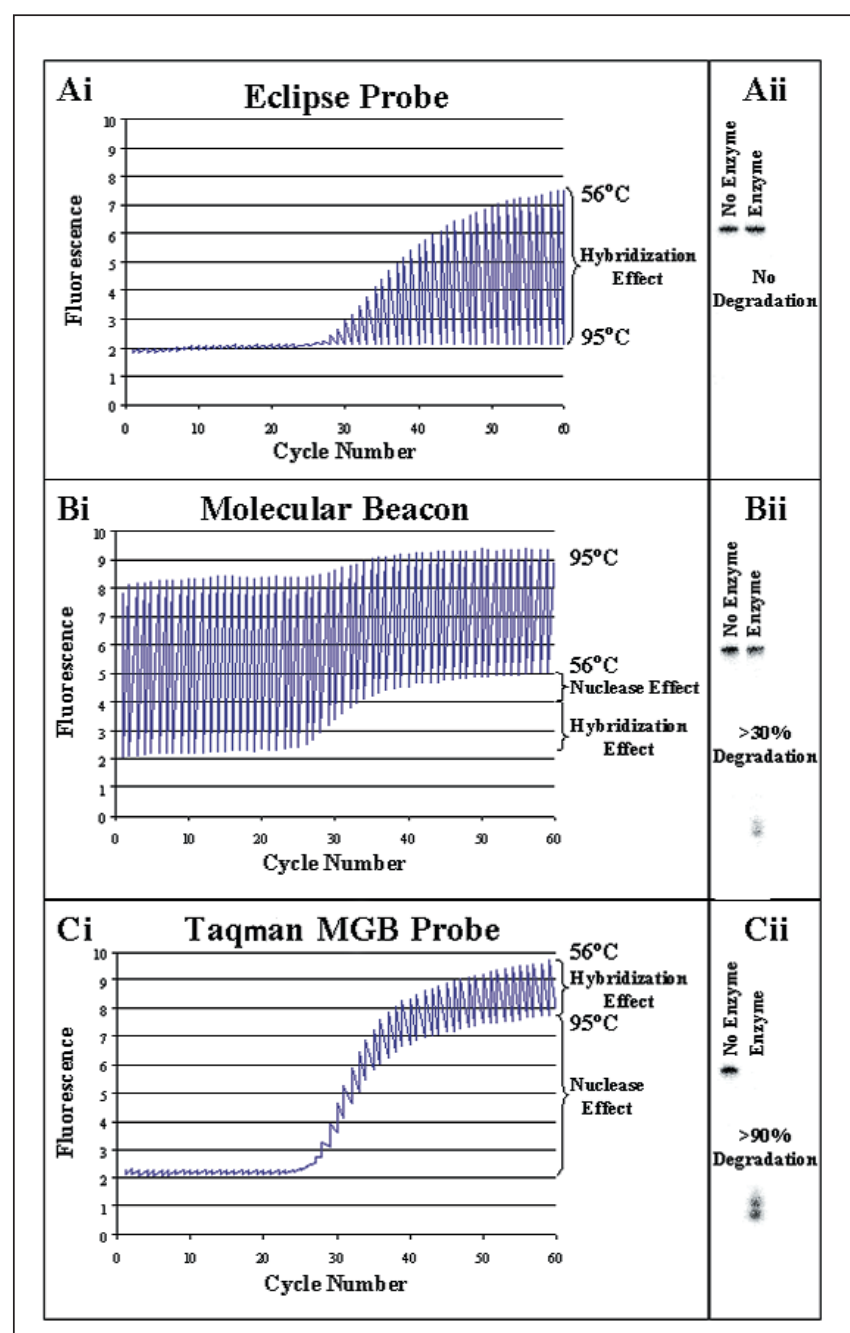

Figure 2. Fluorescent behavior and $5^{\prime}$-nuclease resistance of MGB Eclipse (A), Molecular Beacon (B), and Taqman MGB (C) probes. Realtime PCRs were performed with RRM1 primers and MGB Eclipse (A, i), Molecular Beacon (B, i), and Taqman MGB (C, i) FAM-labeled or ${ }^{32} \mathrm{P}-1 \mathrm{a}-$ beled probes (A, ii; B, ii; C, ii) in the LightCycler, as described with human "66 son" pedigree DNA as a template in the LightCycler. Fluorescent readings were taken at denaturing step $\left(95^{\circ} \mathrm{C}\right)$ and annealing step $\left(56^{\circ} \mathrm{C}\right)$ of the reaction. Control reactions for radioactive probes did not have Taq DNA polymerase (no enzyme lanes). After completion of the PCR, radioactive reactions were subjected to electrophoresis, autoradiographed, and analyzed on a phosphor imager. Respective images are placed to the right of the real-time fluorescent plots. 

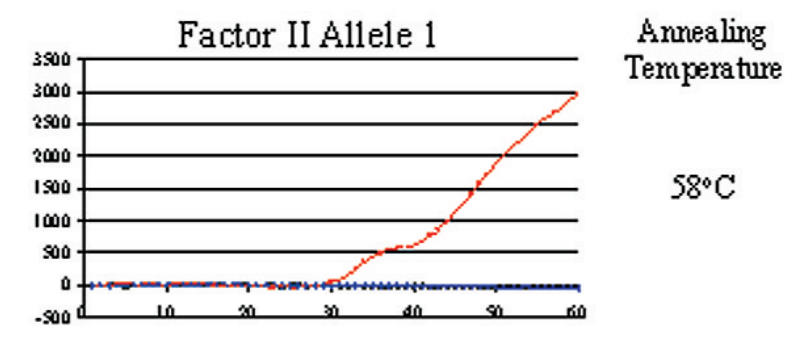

HFE Allele 1
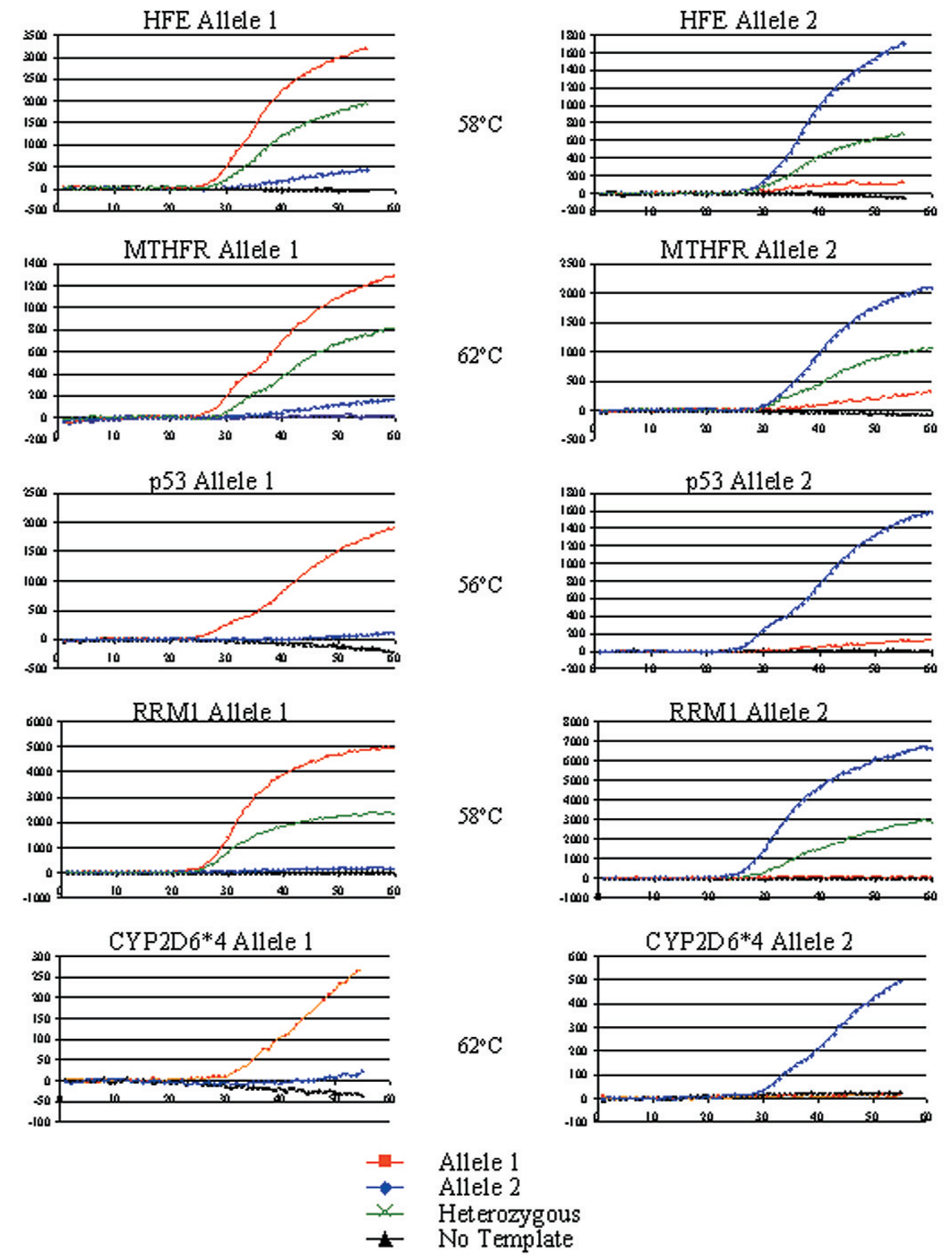

Figure 3. SNP detection with MGB Eclipse probes. PCRs were conducted in an ABI PRISM 7700, as described with both FAM-labeled Allele 1 and TET-labeled Allele 2 MGB Eclipse probes added together (Table 1). Human genomic DNA ( $25 \mathrm{ng}$ ) representing different alleles or $10^{6}$ copies of wild-type or mutant plasmid (p53 mutation) was used in a $25-\mu \mathrm{L}$ reaction. Fluorescent readings were taken at the annealing stage. PCR profiles were as described, with various annealing temperatures indicated by the real-time plots. After PCR, the data were normalized to the signal in cycles $3-15$, which is the default treatment by the ABI PRISM 7700 software. Clipped files were transferred to Microsoft Excel and plotted. 
probes. Table 1 shows the probe and primer sequences. Experiments were conducted in an ABI PRISM 7700, as described in the Materials and Methods section, with both probes added together to the PCR mixture. Annealing temperature (indicated in Figure 3) was optimized for each case to produce the best mismatch discrimination at the highest fluorescent signal. The ABI PRISM 7700 calculates fluorescence emitted by both dyes from a composite spectrum and allows one to view the results in either the FAM or TET channels. Samples that are homozygous for allele 1 show high fluorescence increase in the FAM channel and no fluorescence increase in the TET channel and can be genotyped as allele 1-positive. Samples that are homozygous for allele 2 are the reverse: high fluorescence increase in TET channel and no fluorescence increase in FAM channel. They can be genotyped as allele 2-positive. Heterozygous samples demonstrate a moderate fluorescence increase in both channels. The genotyping is in agreement with RFLP (when possible) and Taqman MGB assays conducted in our laboratory with the same DNA samples.

\section{Viral Load Determination}

An inherent feature of all systems exploiting exponential amplification, such as real-time PCR, is a wide dynamic range (over seven orders of magnitude) and high sensitivity, with the detection limit down to a single copy number (7). To demonstrate that it also applies to the MGB Eclipse system, we titrated DNA fragments containing HSV type 1, human coxsackievirus type A16, Varicella-Zoster virus, Epstein-Barr virus, and a number of other viral sequences to show these properties of MGB Eclipse probes. Typically, a range of $5 \times$ $10^{7}$ to 5 copies/reaction is detected with excellent reproducibility. A representative amplification plot (Figure 4) shows an HSV type 1 titration from $5 \times 10^{6}$ down to 5 copies. The threshold cycle $\left(\mathrm{C}_{t}\right)$ values for these reactions were from 16 to 37 , respectively. When $C_{t}$ was plotted versus the $\operatorname{LogN}$ of the number of input target copies, the resulting standard curve is linear over seven orders of magnitude with a correlation coefficient of 0.999 . Quantitative PCR analysis with MGB Eclipse probes provides a wide linear dynamic range of amplification and sensitivity down to a few copies. This range and sensitivity is as expected for real-time PCR assays (7).

\section{Gene Expression Analysis}

When preceded by an RT of mRNA, real-time PCR is an accurate and convenient method for quantifying gene expression. We evaluated this real-time RT-PCR in both one- and

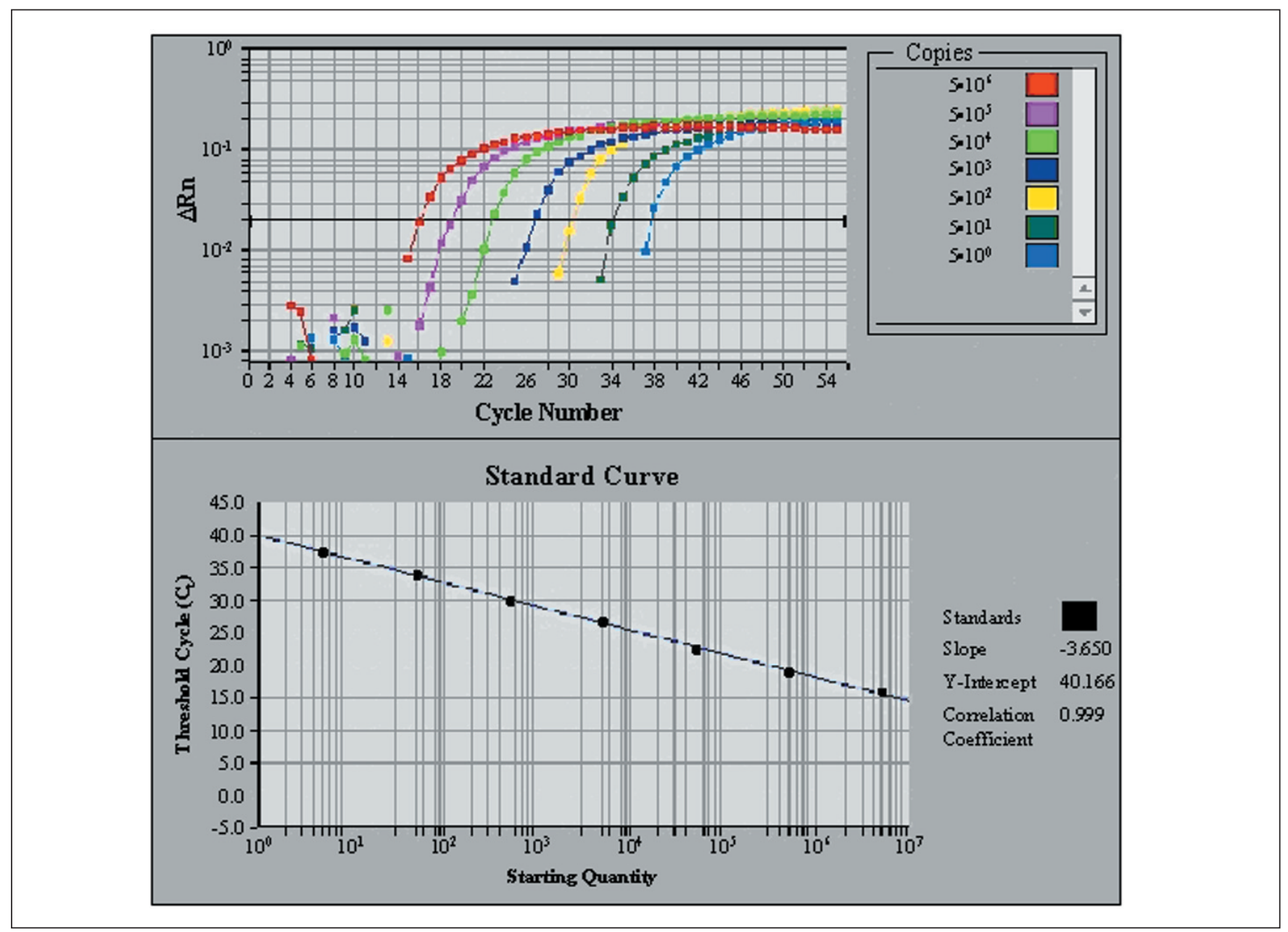

Figure 4. Titration of DNA fragment containing HSV 1 partial viral sequence. Samples of a DNA dilution series, decreasing from $5 \times 10^{6}$ to 5 copies of the HSV 1 partial sequence per reaction, were amplified in an ABI PRISM 7700 with HSV primers and FAM-labeled MGB Eclipse probe as described. The standard curve was generated by the Sequence Detection application of the ABI PRISM 7700. The bottom plot displays the threshold cycle $\left(\mathrm{C}_{\mathrm{t}}\right)$ versus $(\mathrm{LogN})$ starting copy number of each sample. The calculated values for slope, Y-intercept, and correlation coefficient are displayed to the right of the graph. 


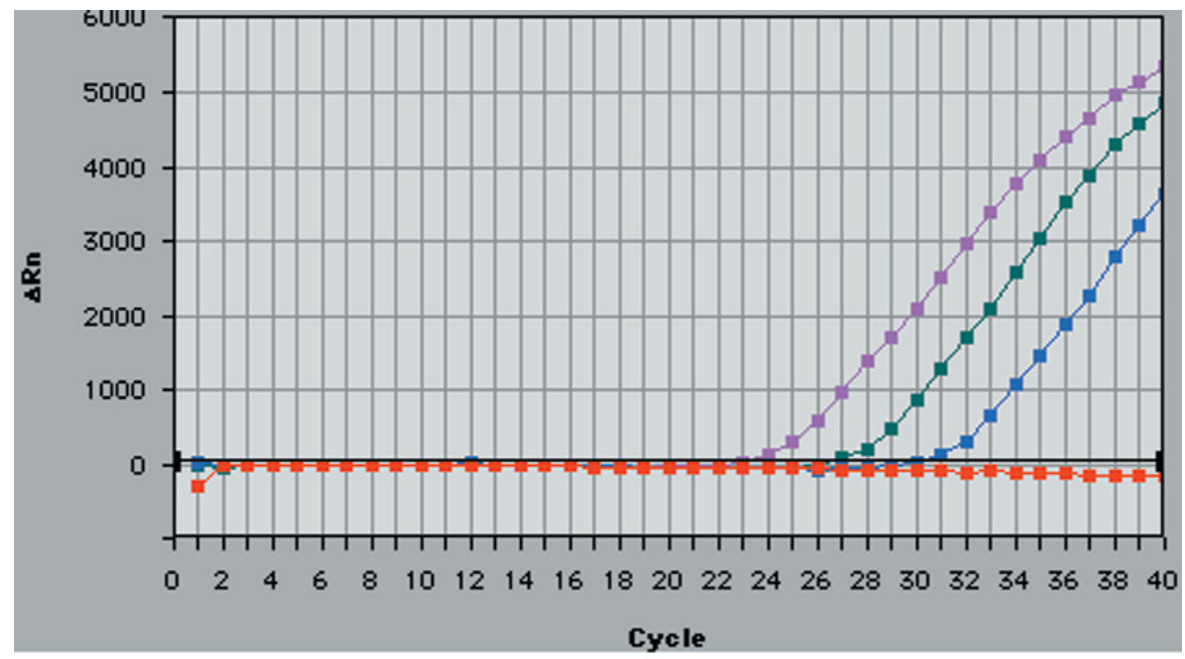

$\square 100$ ng total RNA

$\square 1$ ng total RNA

$\square 10 \mathrm{ng}$ total RNA

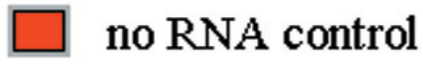

Figure 5. Gene expression analysis of human c-myc protooncogene. RT-PCR was performed with c-myc primers $(0.1 \mu \mathrm{M}$ sense primer, $4 \mu \mathrm{M}$ antisense primer) and $0.2 \mu \mathrm{M}$ sense FAM-labeled MGB Eclipse probe in an ABI PRISM 7700 with the indicated amount of total RNA from human placenta using the OneStep RT-PCR Kit, as recommended in manufacturer's protocol. The annealing temperature was $56^{\circ} \mathrm{C}$, the extension temperature was $76^{\circ} \mathrm{C}$, and the final extension step was omitted. Fluorescent readings were taken at the annealing step $\left(56^{\circ} \mathrm{C}\right)$ of the reaction. 
two-step formats. The OneStep RT-PCR Kit was used for the former, with varying amounts of total RNA from human placenta as a template source. For the two-step RT-PCR, firststrand cDNA synthesis was done using total RNA from human placenta and the Advantage-for-RT-PCR kit. cDNA created in the RT reaction was then used as a template for subsequent amplification.

We have amplified and quantified several human genes in both formats, including $\beta$-actin, c-myc, and c-fos protooncogene. Figure 5 shows a representative one-step real-time RTPCR with different amounts of total RNA, c-myc-specific primers, and FAM-labeled Eclipse probes.

We have previously shown that, under certain conditions, oligonucleotides bearing a $5^{\prime}$-MGB can arrest the extension of DNA polymerase (1). Therefore, to allow efficient PCR, MGB Eclipse probes require an extension step during PCR under conditions that they can be partially melted off the target. We found (data not shown) that extension temperatures in the range of $76^{\circ} \mathrm{C}-80^{\circ} \mathrm{C}$ (depending on the probe stability) are preferable. We have also found that asymmetric PCR facilitates signal increase dramatically. At the start of a reaction, when the probe is in excess, the template and product are at low enough concentrations that product renaturation does not compete with probe binding, allowing amplification to proceed at an exponential rate. With the progression of PCR, the reaction enters a linear phase when product renaturation begins to compete with primer binding. The linear phase in asymmetric PCR produces mostly single-stranded products, making it easier for MGB Eclipse probes to bind without the competition from the complementary strand. The $\mathrm{C}_{\mathrm{t}}$ values determined in the exponential phase are unaffected by primer concentration ratio, but the use of the asymmetric concentrations of primers gives much better end-point fluorescence. Our primer titration experiments show that the best primer ratio range is between 1:5 and 1:40 (data not shown). Reducing the ratio leads to signal reduction, and increasing it does not improve the outcome.

The data presented here demonstrate that real-time PCR with 5'-MGB probes possessing hybridization-triggered fluorescence (MGB Eclipse probes) is a valid alternative to existing techniques, with distinct advantages for a broad spectrum of PCR users. These include the simplicity imparted by short length, robust sensitivity and specificity, and use with a versatile range of fluorophores. Since MGB Eclipse probes are strictly hybridization based, they could be used in amplification systems other than PCR. Among the potential applications could be rolling circle amplification (4), amplification methods based on a thermostable ligase (5), or RNA detection systems like NASBA (3).

\section{ACKNOWLEDGMENTS}

The authors thank Drs. Rich Meyer and Merl Hoekstra for critical review of the manuscript, Drs. Eugene Lukhtanov and Vladimir Gorn for their helpful advice, and Alan Mills for his help with graphical work.

\section{REFERENCES}

1.Afonina, I., I. Kutyavin, E. Lukhtanov, R.B. Meyer, and H. Gamper. 1996. Sequence-specific arrest of primer extension on single-stranded DNA by an oligonucleotide-minor groove binder conjugate. Proc. Natl. Acad. Sci. USA 93:199-204.

2.Afonina, I., M. Zivarts, I. Kutyavin, E. Lukhtanov, H. Gamper, and R.B. Meyer. 1997. Efficient priming of PCR with short oligonucleotides conjugated to a minor groove binder. Nucleic Acids Res. 25:2657-2660.

3.Baeumner, A.J., M.C. Humiston, R.A. Montagna, and R.A. Durst. 2001. Detection of viable oocysts of Cryptosporidium parvum following nucleic acid sequence based amplification. Anal. Chem. 73:1176-1180.

4.Baner, J., M. Nilsson, M. Mendel-Hartvig, and U. Landegren. 1998. Signal amplification of padlock probes by rolling circle replication. Nucleic Acids Res. 26:5073-5078.

5.Barany, F. 1991. Genetic disease detection and DNA amplification using cloned thermostable ligase. Proc. Natl. Acad. Sci. USA 88:189-193.

6.Didenko, V.V. 2001. DNA probes using fluorescence resonance energy transfer (FRET): designs and applications. BioTechniques 31:1106-1121.

7.Foy, C.A. and H.C. Parkers. 2001. Emerging homogeneous DNA-based technologies in the clinical laboratory. Clin. Chem. 47:900-1000.

8.Hurley, L.H., V.L. Reynolds, D.H. Swenson, G.L. Petzold, and T.A. Scahill. 1984. Reaction of the antitumor antibiotic CC-1065 with DNA: structure of a DNA adduct with DNA sequence specificity. Science 226:843-844.

9.Kuimelis, R.G., K.J. Livak, B. Mullah, and A. Andrus. 1997. Structural analogues of TaqMan probes for real-time quantitative PCR. Nucleic Acids Symp. Ser. 37:255-256.

10.Kutyavin, I.V., I.A. Afonina, A. Mills, V.V. Gorn, E.A. Lukhtanov, E.S. Belousov, M.J. Singer, D.K. Walburger et al. 2000. 3'-minor groove binder-DNA probes increase sequence specificity at PCR extension temperatures. Nucleic Acids Res. 28:655-661.

11.Livak, K.J., S.J. Flood, J. Marmaro, W. Giusti, and K. Deetz. 1995. Oligonucleotides with fluorescent dyes at opposite ends provide a quenched probe system useful for detecting PCR product and nucleic acid hybridization. PCR Methods Appl. 4:357-362.

12.Lukhtanov, E.A., I.V. Kutyavin, H.B. Gamper, and R.B. Meyer. 1995. Oligodeoxyribonucleotides with conjugated dihydropyrroloindole oligopeptides: preparation and hybridization properties. Bioconjug. Chem. 6:418-426.

13.Lukhtanov, E.L., M. Metcalf, and M.W. Reed. 2001. Fluorogenic DNA probes for multicolor hybridization assays. Am. Biotechnol. Lab. 19:68-69.

14.Lynas, C. 1997. A cheaper and more rapid polymerase chain reaction-restriction fragment length polymorphism method for the detection of the HLA-H gene mutations occurring in hereditary hemochromatosis. Blood 90:4235-4236.

15.Nazarenko, I.A., S.K. Bhatnagar, and R.J. Hohman. 1997. A closed tube format for amplification and detection of DNA based on energy transfer. Nucleic Acids Res. 25:2516-2521.

16.Nickerson, D.A., R. Kaiser, S. Lappin, J. Stewart, L. Hood, and U. Landegren. 1990. Automated DNA diagnostics using an ELISA-based oligonucleotide ligation assay. Proc. Natl. Acad. Sci. USA 87:8923-8927.

17.Parker, N.J., C.G. Begley, and R.M. Fox. 1991. Human M1 subunit of ribonucleotide reductase: cDNA sequence and expression in stimulated lymphocytes. Nucleic Acids Res. 19:3741.

18.Parkhurst, K.M. and L.J. Parkhurst. 1995. Donor-acceptor distance distributions in a double-labeled fluorescent oligonucleotide both as a single strand and in duplexes. Biochemistry 34:293-300.

19.Powell, K.F., N.E. Anderson, R.W. Frith, and M.C. Croxson. 1990. Noninvasive diagnosis of herpes simplex encephalitis. Lancet 335:357-358.

20.Ririe, K.M., R.P. Rasmussen, and C.T. Wittwer. 1997. Product differentiation by analysis of DNA melting curves during the polymerase chain reaction. Anal. Biochem. 245:154-160.

21.SantaLucia, J. 1998. A unified view of polymer, dumbbell, and oligonucleotide DNA nearest-neighbor thermodynamics. Proc. Natl. Acad. Sci. USA 95:1460-1465.

22.Tyagi, S., D.P. Bratu, and F.R. Kramer. 1998. Multicolor Molecular Beacons for allele discrimination. Nat. Biotechnol. 16:49-53.

23.Uren, E.C., P.D. Johnson, J. Montanaro, and G.L. Gilbert. 1993. Herpes simplex virus encephalitis in pediatrics: diagnosis by detection of antibodies and DNA in cerebrospinal fluid. Pediatr. Infect. Dis. J. 12:1001-1006.

24.Van Straaten, F., R. Muller, T. Curran, C. Van Beveren, and I.M. Verma. 1983. Complete nucleotide sequence of a human c-onc gene: deduced amino acid sequence of the human c-fos protein. Proc. Natl. Acad. Sci. USA 80:3183-3187.

Address correspondence to Dr. Irina Afonina, Epoch Biosciences, 21720-23rd Dr. SE, Suite 150, Bothell, WA 98021, USA.e-mail: iafonina@epochbio.com 